\title{
Development of a Cryo-correlative Light and Electron Microscopy Workflow at Simons Electron Microscopy Center
}

Ashleigh Raczkowski ${ }^{1}$, Kotaro Kelley ${ }^{1}$, Alex Noble ${ }^{1}$, Edward Eng ${ }^{1}$, Micah Rapp ${ }^{2}$, Mykhailo Kopylov ${ }^{1}$, Maryam Sayyahmanesh ${ }^{1}$, Clint Potter $^{1}$ and Bridget Carragher ${ }^{1}$

${ }^{1}$ New York Structural Biology Center, New York, New York, United States, ${ }^{2}$ Columbia University, New York, New York, United States

Cryo-electron tomography (Cryo-ET) has become a robust method for visualizing cellular and molecular structures within their native environment (Beck \& Baumeister, 2016). Biological samples are vitrified by plunging into liquid nitrogen-cooled liquid ethane followed by sample preparation and tilt-series acquisition in a transmission electron microscope (TEM). However, most biological samples in native contexts are too thick for cryo-ET collection in a TEM. The mean free path of a single electron scattering event at $300 \mathrm{kV}$ limits the thickness of a specimen that can be imaged in a TEM to less than $300 \mathrm{~nm}$. By utilizing a focused ion beam-scanning electron microscope (FIB-SEM) at cryogenic temperatures as a tool for micro machining of cells and bulk samples, these thickness limitations can be surpassed by milling thin windows, or lamellas, into cells that are then thin enough to be imaged in a TEM. Specimen thickness is not only problematic during image acquisition, but also during the vitrification process. For samples frozen using commercial plunge freezers, the thickness limit is about 5-10 $\mu \mathrm{m}$ in order to ensure the entire cell has been vitrified. The use of high-pressure freezing (HPF) enables the vitrification of much thicker samples into the hundreds of microns. We are currently in the process of developing a general method we call the 'waffle method' which leverages high-pressure freezing for addressing several challenges, including milling specimen with preferred orientation, low throughput when milling small specimen, cellular specimen that concentrate poorly in grid squares, and thick specimen with vitrification issues, among other challenges. The introduction of a cryo correlative light and electron microscopy (CLEM) workflow has enabled the localization of fluorescently-tagged proteins of interest within the cell for more optimized FIB-milling and targeting during tomographic data collection. Here we present our developing cryo-CLEM/cryo-FIB-SEM/cryo-ET pipeline with both high pressure frozen and plunge frozen samples.

\section{References}

Beck, M., \& Baumeister, W. (2016). Cryo-Electron Tomography: Can it Reveal the Molecular Sociology of Cells in Atomic Detail? Trends in Cell Biology, 26(11), 825-837. doi:10.1016/j.tcb.2016.08.006 